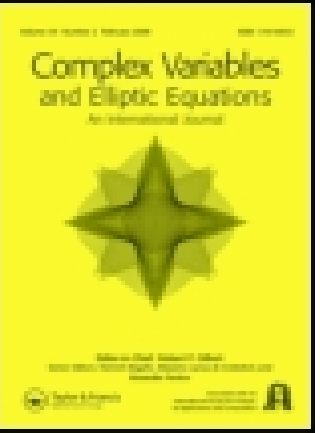

Complex Variables

ISSN: 0278-1077 (Print) 1563-5066 (Online) Journal homepage: https://www.tandfonline.com/loi/gcov19

\title{
Distance between a Maximum Modulus Point and Zero Set of an Entire Function
}

\section{lossif Ostrovskii \& Adem Ersin Üreyen}

To cite this article: Iossif Ostrovskii \& Adem Ersin Üreyen (2003) Distance between a Maximum Modulus Point and Zero Set of an Entire Function, Complex Variables, 48:7, 583-598, DOI: 10.1080/0278107031000120431

To link to this article: https://doi.org/10.1080/0278107031000120431

册 Published online: 15 Sep 2010.

Submit your article to this journal $\pi$

山 Article views: 21

Citing articles: 2 View citing articles $\square$ 


\title{
Distance between a Maximum Modulus Point and Zero Set of an Entire Function
}

\author{
IOSSIF OSTROVSKII ${ }^{a, b, *}$ and ADEM ERSIN ÜREYEN ${ }^{a}$ \\ ${ }^{a}$ Department of Mathematics, Bilkent University, 06800 Bilkent, Ankara, Turkey; \\ berkin Institute of Low Temperature Physics and Engineering, 61103 Kharkov, Ukraine
}

Communicated by M. Essen

(Received 6 December 2002; In final form 15 March 2003)

Let $f$ be an entire function of finite positive order. A maximum modulus point is a point $w$ such that $|f(w)|=\max \{|f(z)|:|z|=|w|\}$. We obtain lower bounds for the distance between a maximum modulus point $w$ and the zero set of $f$. These bounds are valid for all sufficiently large values of $|w|$.

Keywords: Entire function; Order; Proximate order; Strong proximate order; Type

2000 AMS Subject Classification: 30D20

\section{INTRODUCTION}

Let $f$ be an entire function. A point $w \in \mathbb{C}$ is called a maximum modulus point if

$$
|f(w)|=M(|w|, f),
$$

where

$$
M(r, f)=\max _{|z|=r}|f(z)|
$$

We denote by $R(w, f)$ the distance between a maximum modulus point $w$ and the zero set of $f$, i.e.

$$
R(w, f)=\inf \{|w-z|: f(z)=0\} .
$$

\footnotetext{
*Corresponding author. E-mail: iossif@fen.bilkent.edu.tr; OSTROVSKII@ilt.kharov.ua
} 
Following Macintyre [3], let us introduce the nondecreasing function

$$
K(r, f)=\frac{d}{d \log r} \log M(r, f), \quad r>0,
$$

(we take the right derivative at fracture points of $\log M(r, f)$ ).

Macintyre [3] showed that the well-known Wiman-Valiron formula (see, e.g. [1], p.22) describing the behavior of $f$ in a neighborhood of a maximum modulus point remains valid with $K(r, f)$ instead of the central index of $f$. Namely, Macintyre showed that

$$
f(z)=f(w)\left(\frac{z}{w}\right)^{K(|w|, f)}(1+o(1)), w \rightarrow \infty, \quad|w| \notin E,
$$

where $E \subset(0, \infty)$ is an exceptional set and $z$ lies in a (depending on $w$ ) neighborhood of $w$.

In Macintyre's proof of formula (1) estimates of $R(w, f)$ from below played an important role. We formulate these estimates as the following separate theorem.

THEOREM A ([3])

(i) The following inequality holds

$$
\limsup _{|w| \rightarrow \infty} \frac{1}{|w|} R(w, f)(\log M(|w|, f))^{1 / 2}>0 .
$$

(ii) For each $\epsilon>0$ the following inequality holds

$$
\liminf _{\substack{|w| \rightarrow \infty \\|w| \notin A_{\epsilon}}} \frac{1}{|w|} R(w, f)(\log M(|w|, f))^{(1 / 2)+\epsilon}>0,
$$

where $A_{\epsilon} \subset \mathbb{R}_{+}$is such that

$$
\int_{A_{\epsilon}} \frac{d t}{t}<\infty
$$

The inequality (2) gives an asymptotic bound for $R(w, f)$ from below only on $a$ sequence of values of $|w| \rightarrow \infty$. The inequality (3) gives a bit less precise bound which is valid outside of a "small" set. In this article we are going to show that bounds for $R(w, f)$ given by (2) and (3) cannot be valid in general without exceptional sets at all and find in some sense unimprovable bounds valid for all sufficiently large values of $|w|$.

Note that far reaching generalizations of (1)-(3) to functions analytic in a half-plane, multi-valued functions and entire functions of several variables were obtained by Sh. Strelitz [6]. We think that similar generalizations are possible for our results as well, but we do not touch them here and restrict ourselves to entire functions of one variable and finite positive order.

Note also that, for some specific parametrical families $\left\{f_{\alpha}\right\}, 0<\alpha<\infty$, of entire functions of order 1 and type $\alpha$ (so called "grand partition functions"), bounds 
for $R\left(w, f_{\alpha}\right)$ from below play an important role in the theory of phase transitions (see [5], Chap. 3). Nevertheless, we cannot extract from our results any useful consequences for this theory because our approach does not permit to take into account asymptotical behavior in parameter $\alpha \rightarrow \infty$.

\section{STATEMENT OF RESULTS}

Let $f$ be an entire function of order $\rho$. We assume that $0<\rho<\infty$.

To state our results we need the notions of proximate order ([4], p.31) and strong proximate order ([4], p.41).

Remind that a proximate order is a function $\rho(r) \in C^{1}\left(\mathbb{R}_{+}\right)$such that

(i) $\exists \lim _{r \rightarrow \infty} \rho(r)=\rho(>0)$,

(ii) $\lim _{r \rightarrow \infty} \rho^{\prime}(r) r \log r=0$.

Note that $([4], \mathrm{p} .32)$

$$
\lim _{r \rightarrow \infty} r^{-\rho(r)}(k r)^{\rho(k r)}=k^{\rho}
$$

uniformly on each interval $0<a \leq k \leq b<\infty$.

By Valiron's theorem ([4], p.35) any entire function $f$ of order $\rho$ has its own proximate order, that is there exists such a proximate order $\rho(r) \rightarrow \rho$ that the type

$$
\sigma:=\limsup _{r \rightarrow \infty} r^{-\rho(r)} \log M(r, f)
$$

is finite and positive. We denote by $[\rho(r), \sigma]$ the set of all entire functions having proximate order $\rho(r)$ and type $\sigma$.

Strong proximate order is a function $\rho^{*}(r) \in C^{2}\left(\mathbb{R}_{+}\right)$representable in the form

$$
\rho^{*}(r)=\rho+\frac{\vartheta_{2}(\log r)-\vartheta_{1}(\log r)}{\log r}
$$

where $\vartheta_{1}$ and $\vartheta_{2}$ are concave functions on $\mathbb{R}$ satisfying conditions $(i=1,2)$ :

(i) $\lim _{x \rightarrow \infty} \vartheta_{i}(x)=\infty$;

(ii) $\lim _{x \rightarrow \infty} \vartheta_{i}(x) / x=0$;

(iii) $\lim _{x \rightarrow \infty} \vartheta_{i}^{\prime \prime}(x) / \vartheta_{i}^{\prime}(x)=0$.

Note that these properties imply

$$
\lim _{x \rightarrow \infty} \vartheta_{i}^{\prime}(x)=\lim _{x \rightarrow \infty} \vartheta_{i}^{\prime \prime}(x)=0, \quad i=1,2
$$

By Levin's theorem ([4], p.39), any entire function of order $\rho$ has its own strong proximate order (6), that is such a strong proximate order $\rho^{*}(r) \rightarrow \rho$ that the type

$$
\sigma:=\limsup _{r \rightarrow \infty} r^{-\rho^{*}(r)} \log M(r, f)
$$


is finite and positive. We denote by $\left[\rho^{*}(r), \sigma\right]$ the set of all entire functions of strong proximate order $\rho^{*}(r)$ and type $\sigma$.

Our first result is the following.

THEOREM 1

(i) If $f \in[\rho(r), \sigma]$, then

$$
\liminf _{|w| \rightarrow \infty}|w|^{\rho(|w|)-1} R(w, f) \geq\left(e^{2} \rho \sigma\right)^{-1} .
$$

(ii) If $f \in[\rho(r), \sigma]$ has nonnegative Taylor coefficients, then

$$
\liminf _{|w| \rightarrow \infty}|w|^{\rho(|w|)-1} R(|w|, f) \geq(e \rho \sigma)^{-1} .
$$

(iii) There exists $f \in[\rho(r), \sigma]$ such that

$$
\liminf _{|w| \rightarrow \infty}|w|^{\rho(|w|)-1} R(w, f) \leq \pi(e \rho \sigma)^{-1} .
$$

We do not know whether the constants $\left(e^{2} \rho \sigma\right)^{-1}$ and $(e \rho \sigma)^{-1}$ in the right hand sides of (8) and (9) are the best possible. Nevertheless, part (iii) of Theorem 1 shows that the best possible constant is not greater than $\pi(e \rho \sigma)^{-1}$.

Let us compare Theorem 1 with Macintyre's Theorem A. It is easy to see that, for $f \in[\rho(r), \sigma]$, Theorem A implies

(i') For some sequence of $w$ tending to $\infty$,

$$
R(w, f)>C|w|^{1-\rho(|w|) / 2} .
$$

(ii') For $|w| \notin A_{\epsilon}$, where $A_{\epsilon} \subset \mathbb{R}_{+}$satisfies (4),

$$
R(w, f)>C|w|^{1-\epsilon-\rho(|w|) / 2} .
$$

Here $C$ denotes a positive constant.

Part (i) of Theorem 1 shows that $R(w, f)>C|w|^{1-\rho(|w|)}$. This estimate is less precise than (11) and (12), but it is valid for all $w$. Moreover, part (iii) of Theorem 1 shows that Macintyre's estimates (11) and (12) cannot be valid for all $w$.

If we consider functions of regular growth, then we can get a better bound for $R(w, f)$, than (8).

Theorem 2 Let $f \in\left[\rho^{*}(r), \sigma\right]$. Assume

$$
\log M(r, f)=\sigma r^{\rho^{*}(r)}+O(\psi(r)), \quad r \rightarrow \infty,
$$

where $\psi(r)>0$ is a nondecreasing function such that

$$
\begin{aligned}
& \text { (i) } \psi(r)=o\left(r^{\rho^{*}(r)}\right), \quad r \rightarrow \infty, \\
& \text { (ii) } \psi(2 r)=O(\psi(r)), \quad r \rightarrow \infty,
\end{aligned}
$$


then

$$
\liminf _{|w| \rightarrow \infty}|w|^{\rho^{*}(|w|)-1} R(w, f) \sqrt{\frac{\psi(|w|)}{|w|^{\rho^{*}(|w|)}}}>0 .
$$

It is easy to see that (15) is a better estimate than (8). Moreover, the smaller the function $\psi$ is the better the bound for $R(w, f)$ is. In particular, if $\psi(r)=O(1), r \rightarrow \infty$, then $R(w, f)>C|w|^{1-\rho^{*}(|w|) / 2}$, i.e. Macintyre's bound (11) remains valid for all $w$ in this case.

In general, the bound (15) cannot be improved as the example of the Weierstrass sigma-function with lattice consisting of integer points of $\mathbb{C}$ shows. In this example, (13) holds with $\rho^{*}(r) \equiv 2, \sigma=\pi / 4, \psi(r) \equiv 1$ (see [2], p.346) and, evidently, $R(w, f) \leq 1 / \sqrt{2}$.

\section{PROOF OF THEOREM 1}

Without loss of generality let us assume that $f(0)=1$. We need the following lemma. LEMma 3.1 The following inequality holds

$$
\limsup _{r \rightarrow \infty} \frac{K(r, f)}{r^{\rho(r)}} \leq e \rho \sigma .
$$

Proof For any $k \in(1, \infty)$ we have

$$
\begin{aligned}
\log M(k r, f) & =\int_{0}^{k r} \frac{d}{d t} \log M(t, f) d t=\int_{0}^{k r} \frac{K(t, f)}{t} d t \geq \int_{r}^{k r} \frac{K(t, f)}{t} d t \\
& \geq K(r, f) \int_{r}^{k r} \frac{d t}{t}=K(r, f) \log k .
\end{aligned}
$$

Hence,

$$
\limsup _{r \rightarrow \infty} \frac{K(r, f)}{r^{\rho(r)}} \leq \frac{1}{\log k} \limsup _{r \rightarrow \infty} \frac{\log M(k r, f)}{(k r)^{\rho(k r)}} \frac{(k r)^{\rho(k r)}}{r^{\rho(r)}} .
$$

Using (5), we get

$$
\limsup _{r \rightarrow \infty} \frac{K(r, f)}{r^{\rho(r)}} \leq \frac{k^{\rho} \sigma}{\log k}
$$

Taking minimum with respect to $k>1$ in the right hand side, we obtain (16).

Now we will prove part (i) of Theorem 1. Let $w$ be a maximum modulus point of $f$. Consider the function

$$
\Phi_{w}(z):=\frac{f(w+z)}{f(w)} .
$$


Let

$$
Q(h, w):=\max _{|z| \leq h}\left|\Phi_{w}(z)\right|
$$

Evidently, $Q(h, w)>1$ for $h>0$ because $\Phi_{w}(0)=1$. Since $|f(w+z)| \leq M(|w|+|z|, f)$, we have

$$
Q(h, w) \leq \frac{M(|w|+h, f)}{M(|w|, f)}
$$

Hence,

$$
\begin{aligned}
\log Q(h, w) & \leq \log M(|w|+h, f)-\log M(|w|, f)=\int_{|w|}^{|w|+h} \frac{d}{d t} \log M(t, f) d t \\
& =\int_{|w|}^{|w|+h} \frac{K(t, f)}{t} d t \leq K(|w|+h, f) \int_{|w|}^{|w|+h} \frac{d t}{t} \\
& =K(|w|+h, f) \log \left(1+\frac{h}{|w|}\right) \leq K(|w|+h, f) \frac{h}{|w|} .
\end{aligned}
$$

By Lemma 3.1, for each $\epsilon>0$ there exists $r_{\epsilon}$ such that $K(r, f) \leq(e \rho \sigma+\epsilon) r^{\rho(r)}$ for $r>r_{\epsilon}$. Hence,

$$
\log Q(h, w) \leq(e \rho \sigma+\epsilon)(|w|+h)^{\rho(|w|+h)} \frac{h}{|w|}, \quad \text { for }|w|>r_{\epsilon} .
$$

Following Macintyre [3] consider the function

$$
\eta_{w}(z):=\frac{Q\left(\Phi_{w}(z)-1\right)}{Q^{2}-\Phi_{w}(z)}
$$

where $Q=Q(h, w)$. Using properties of bilinear transformation and taking into account that by definition of $Q$, we have $\left|\Phi_{w}(z)\right| \leq Q$ for $|z| \leq h$, we conclude that $\left|\eta_{w}(z)\right| \leq 1$ for $|z| \leq h$. Since $\eta_{w}(0)=0$, Schwarz lemma implies

$$
\left|\eta_{w}(z)\right| \leq|z| / h, \quad \text { for }|z| \leq h .
$$

Hence,

$$
Q\left|\Phi_{w}(z)-1\right| \leq \frac{|z|}{h}\left|Q^{2}-\Phi_{w}(z)\right| \leq \frac{|z|}{h}\left(\left|Q^{2}-1\right|+\left|\Phi_{w}(z)-1\right|\right) .
$$

Thus,

$$
\left|\Phi_{w}(z)-1\right| \leq \frac{(|z| / h)\left(Q^{2}-1\right)}{Q-|z| / h} .
$$


Since

$$
\frac{(|z| / h)\left(Q^{2}-1\right)}{Q-|z| / h}<1, \quad \text { for }|z|<h / Q
$$

we get

$$
\left|\Phi_{w}(z)-1\right|<1, \quad \text { for }|z|<h / Q \text {. }
$$

Hence,

$$
\Phi_{w}(z) \neq 0, \quad \text { for }|z|<h / Q
$$

and therefore $f(w+z) \neq 0$. This implies

$$
R(w, f) \geq h / Q
$$

Using (17), we obtain

$$
R(w, f) \geq h \exp \left\{-(e \rho \sigma+\epsilon) \frac{h}{|w|}(|w|+h)^{\rho(|w|+h)}\right\}, \quad|w|>r_{\epsilon} .
$$

Setting

$$
h=(e \rho \sigma)^{-1}|w|^{1-\rho(|w|)},
$$

we get

$$
R(w, f) \geq \frac{|w|^{1-\rho(|w|)}}{e \rho \sigma} \exp \left\{-\frac{(e \rho \sigma+\epsilon)}{e \rho \sigma} \frac{(|w|+h)^{\rho(|w|+h)}}{|w|^{\rho(|w|)}}\right\} .
$$

Hence,

$$
\liminf _{|w| \rightarrow \infty}|w|^{\rho(|w|)-1} R(w, f) \geq \liminf _{|w| \rightarrow \infty} \frac{1}{e \rho \sigma} \exp \left\{-\frac{(e \rho \sigma+\epsilon)}{e \rho \sigma} \frac{(|w|+h)^{\rho(|w|+h)}}{|w|^{\rho(|w|)}}\right\} .
$$

Since (5) holds uniformly in $k$ on any interval $0<a \leq k \leq b<\infty$, we obtain

$$
\lim _{|w| \rightarrow \infty} \frac{(|w|+h)^{\rho(|w|+h)}}{|w|^{\rho(|w|)}}=\lim _{|w| \rightarrow \infty} \frac{(|w|(1+h /|w|))^{\rho(|w|(1+h /|w|))}}{|w|^{\rho(|w|)}}=1 .
$$

Thus, inequality (18) reduces to

$$
\liminf _{|w| \rightarrow \infty}|w|^{\rho(|w|)-1} R(w, f) \geq \frac{1}{e \rho \sigma} \exp \left\{-\left(1+\frac{\epsilon}{e \rho \sigma}\right)\right\} .
$$

Since this is true for each $\epsilon>0$, we get (8). 
Proof of (ii) Let $f \not \equiv 0$,

$$
f(z)=\sum_{k=0}^{\infty} a_{k} z^{k}, \quad a_{k} \geq 0, k=0,1, \ldots
$$

Then the set of maximum modulus points contains $\mathbb{R}_{+}$. If it does not coincide with $\mathbb{R}_{+}$, then there is $w \notin \mathbb{R}_{+}$such that

$$
\left|\sum_{k=0}^{\infty} a_{k} w^{k}\right|=\sum_{k=0}^{\infty} a_{k}|w|^{k}
$$

This equality may hold if and only if

$$
a_{k} \neq 0 \Rightarrow \arg \left(w^{k}\right) \equiv 0(\bmod 2 \pi) .
$$

It follows that there is an integer $n \geq 2$ such that $a_{k}=0$ for each $k$ being not integer multiple of $n$. Let us take the largest $n$ with this property. Then we have $f\left(z e^{2 \pi i / n}\right)=$ $f(z)$ and the set of maximum modulus points consists of the system of rays $\{z: \arg z=2 \pi j / n\}, j=0, \ldots, n-1$. Therefore without loss of generality we can consider further only maximum modulus points $w$ lying on $\mathbb{R}_{+}$.

Let $z=r e^{i \varphi_{r}},\left|\varphi_{r}\right|<\pi$, be a zero of $f$. We have

$$
f(r)=f(r)-f\left(r e^{i \varphi_{r}}\right)=\sum_{k=0}^{\infty} a_{k} r^{k}\left(1-e^{i k \varphi_{r}}\right) \leq\left|\varphi_{r}\right| \sum_{k=0}^{\infty} k a_{k} r^{k}=\left|\varphi_{r}\right| r f^{\prime}(r) .
$$

Whence

$$
\left|\varphi_{r}\right| \geq \frac{f(r)}{r f^{\prime}(r)}=\frac{1}{K(r, f)}
$$

Assume that (9) is wrong. Then there exists a sequence $0<w_{k} \rightarrow \infty$ and a number $\epsilon>0$ such that

$$
R\left(w_{k}, f\right) \leq(e \rho \sigma+\epsilon)^{-1} w_{k}^{1-\rho\left(w_{k}\right)}=o\left(w_{k}\right), \quad k \rightarrow \infty .
$$

By the definition of $R$, there are zeros $z_{k}=r_{k} e^{i \varphi_{k}}$ of $f$ such that $R\left(w_{k}, f\right)=\left|z_{k}-w_{k}\right|$. Note that (19) implies $r_{k}=(1+o(1)) w_{k}, \varphi_{k}=o(1)$. Hence,

$$
R\left(w_{k}, f\right) \geq\left|\Im z_{k}\right|=r_{k}\left|\varphi_{k}\right|(1+o(1)) \geq \frac{w_{k}}{K\left(r_{k}, f\right)}(1+o(1)) .
$$

This and (19) imply

$$
K\left(r_{k}, f\right) \geq \frac{w_{k}}{R\left(w_{k}, f\right)}(1+o(1)) \geq(e \rho \sigma+\epsilon) w_{k}^{\rho\left(w_{k}\right)}(1+o(1)) .
$$


Hence,

$$
\limsup _{r \rightarrow \infty} \frac{K(r, f)}{r^{\rho(r)}} \geq(e \rho \sigma+\epsilon) \limsup _{k \rightarrow \infty} \frac{w_{k}^{\rho\left(w_{k}\right)}}{r_{k}^{\rho\left(r_{k}\right)}} .
$$

Since $w_{k}=r_{k}(1+o(1))$, (5) implies that the right hand side of (20) equals $e \rho \sigma+\epsilon$ and we obtain a contradiction with (16).

Proof of (iii) Set

$$
f(z)=\prod_{k=1}^{\infty}\left(1+\left(\frac{z}{p_{k}}\right)^{\left[a p_{k}^{\rho\left(p_{k}\right)}\right]}\right)
$$

where

$$
p_{k}=e^{2^{k}}, a=e \rho \sigma .
$$

We should show:

(a) $f \in[\rho(r), \sigma]$

(b) $\liminf _{|w| \rightarrow \infty}|w|^{\rho(|w|)-1} R(w, f) \leq \pi(e \rho \sigma)^{-1}$.

Let us first show (b). It is evident from definition (21) that $f$ has nonnegative Taylor coefficients. Hence $\mathbb{R}_{+}$consists of maximum modulus points of $f$ and in particular, each point $p_{k}$ is such a point. Zeros of $f$ are located at the points,

$$
p_{k} \exp \left\{i \pi(1+2 j) /\left[a p_{k}^{\rho\left(p_{k}\right)}\right]\right\}, \quad j=0,1, \ldots,\left[a p_{k}^{\rho\left(p_{k}\right)}\right]-1, \quad k=1,2, \ldots
$$

Evidently,

$$
R\left(p_{k}, f\right) \leq\left|p_{k}-p_{k} \exp \left\{i \pi /\left[a p_{k}^{\rho\left(p_{k}\right)}\right]\right\}\right|=2 p_{k} \sin \frac{\pi}{2\left[a p_{k}^{\rho\left(p_{k}\right)}\right]}
$$

Therefore,

$$
p_{k}^{\rho\left(p_{k}\right)-1} R\left(p_{k}, f\right) \leq 2 p_{k}^{\rho\left(p_{k}\right)} \sin \frac{\pi}{2\left[a p_{k}^{\rho\left(p_{k}\right)}\right]} .
$$

Taking limit as $k \rightarrow \infty$ and remembering that $a=e \rho \sigma$, we obtain (b).

For part (a), we need to show that

$$
\limsup _{r \rightarrow \infty} r^{-\rho(r)} \log M(r, f)=\sigma .
$$

Let us first prove that the following relation holds

$$
\log M(r, f)=\left[a p_{n}^{\rho\left(p_{n}\right)}\right] \log \frac{r}{p_{n}}+o\left(r^{\rho(r)}\right), \quad r \rightarrow \infty, \quad p_{n}<r \leq p_{n+1} .
$$


We have

$$
\begin{aligned}
\log M(r, f)= & \sum_{k=1}^{\infty} \log \left(1+\left(\frac{r}{p_{k}}\right)^{\left[a p_{k}^{\rho\left(p_{k}\right)}\right]}\right) \\
= & {\left[a p_{n}^{\rho\left(p_{n}\right)}\right] \log \frac{r}{p_{n}}+\sum_{k=1}^{n-1}\left[a p_{n}^{\rho\left(p_{k}\right)}\right] \log \left(\frac{r}{p_{k}}\right) } \\
& \quad+\sum_{k=1}^{n} \log \left(1+\left(\frac{p_{k}}{r}\right)^{\left[a p_{n}^{\rho\left(p_{k}\right)}\right]}\right)+\sum_{k=n+1}^{\infty} \log \left(1+\left(\frac{r}{p_{k}}\right)^{\left[a p_{n}^{\rho\left(p_{k}\right)}\right]}\right) \\
= & :\left[a p_{n}^{\rho\left(p_{n}\right)}\right] \log \frac{r}{p_{n}}+S_{1}+S_{2}+S_{3} .
\end{aligned}
$$

It is easy to show that

$$
S_{1}=o\left(r^{\rho(r)}\right), \quad S_{2}=O(1), S_{3}=O(1), \quad r \rightarrow \infty, \quad p_{n}<r \leq p_{n+1},
$$

which proves (23); we use that $\rho(r) \rightarrow \rho>0$ as $r \rightarrow \infty$ (details omitted).

Now let us prove (22), or equivalently,

$$
\limsup _{r \rightarrow \infty} r^{-\rho(r)} \log M(r, f)=a / e \rho .
$$

Denoting the left hand side of (24) by $\bar{\sigma}$ and using (23), we obtain

$$
\begin{aligned}
\bar{\sigma} & =\limsup _{\substack{r \rightarrow \infty \\
r \in\left(p_{n}, p_{n+1}\right]}} r^{-\rho(r)}\left\{\left[a p_{n}^{\rho\left(p_{n}\right)}\right] \log \left(r / p_{n}\right)+o\left(r^{\rho(r)}\right)\right\} \\
& \leq \limsup _{\substack{r \rightarrow \infty \\
r \in\left(p_{n}, p_{n+1}\right]}} r^{-\rho(r)}\left\{a p_{n}^{\rho\left(p_{n}\right)} \log \left(r / p_{n}\right)\right\} \\
& \leq a \limsup _{n \rightarrow \infty} p_{n}^{\rho\left(p_{n}\right)} \max _{r \in\left(p_{n}, p_{n+1}\right]} g_{n}(r)
\end{aligned}
$$

where $g_{n}(r)=r^{-\rho(r)} \log \left(r / p_{n}\right)$.

Let us find an upper bound of $g_{n}$ for $p_{n}<r \leq p_{n+1}$. We have

$$
g_{n}^{\prime}(r)=r^{-\rho(r)-1}\left[-\left(r \rho^{\prime}(r) \log r+\rho(r)\right) \log \left(r / p_{n}\right)+1\right]
$$

Since $r \rho^{\prime}(r) \log r+\rho(r) \rightarrow \rho$ as $r \rightarrow \infty$, we have for large $n$

$$
r^{-\rho(r)-1}\left[-(\rho+\epsilon) \log \left(r / p_{n}\right)+1\right]<g_{n}^{\prime}(r)<r^{-\rho(r)-1}\left[-(\rho-\epsilon) \log \left(r / p_{n}\right)+1\right],
$$

where $\epsilon$ is an arbitrary number from $(0, \rho)$.

When $r<p_{n} e^{1 /(\rho+\epsilon)}$, the left inequality in (26) implies that $g_{n}^{\prime}(r)>0$. When $r>p_{n} e^{1 /(\rho-\epsilon)}$, the right inequality in (26) implies $g_{n}^{\prime}(r)<0$. Hence, $g_{n}$ attains its 
maximum value on $\left(p_{n}, p_{n+1}\right]$ at some point $r_{n} \in\left[p_{n} e^{1 /(\rho+\epsilon)}, p_{n} e^{1 /(\rho-\epsilon)}\right]$. Therefore, using (5) and (25), we obtain

$$
\begin{aligned}
\bar{\sigma} & \leq a \limsup _{n \rightarrow \infty} p_{n}^{\rho\left(p_{n}\right)} g_{n}\left(r_{n}\right)=a \limsup _{n \rightarrow \infty} \frac{p_{n}^{\rho\left(p_{n}\right)} \log \left(r_{n} / p_{n}\right)}{r_{n}^{\rho\left(r_{n}\right)}} \\
& \leq a \limsup _{n \rightarrow \infty} \frac{p_{n}^{\rho\left(p_{k}\right)}}{\left(p_{n} e^{1 /(\rho+\epsilon)}\right)^{\rho\left(p_{n} e^{1 /(\rho+\epsilon)}\right)}} \frac{1}{\rho-\epsilon}=\frac{a}{e^{\rho /(\rho+\epsilon)}} \frac{1}{(\rho-\epsilon)} .
\end{aligned}
$$

Letting $\epsilon \rightarrow 0$, we get $\bar{\sigma} \leq a / e \rho$.

For the reverse inequality, let $s_{n}=p_{n} e^{1 / \rho}$. Since $\lim _{r \rightarrow \infty} p_{n+1} / p_{n}=\infty$, we have $p_{n}<s_{n} \leq p_{n+1}$ for sufficiently large $n$. Therefore, using (5), we obtain

$$
\begin{aligned}
\bar{\sigma} & \geq \limsup _{n \rightarrow \infty} \frac{\left(a p_{n}^{\rho\left(p_{n}\right)}-1\right) \log \left(s_{n} / p_{n}\right)}{r_{n}^{\rho\left(r_{n}\right)}} \\
& =a \limsup _{n \rightarrow \infty} \frac{p_{n}^{\rho\left(p_{n}\right)}}{\left(p_{n} e^{1 / \rho}\right)^{\rho\left(p_{n} e^{1 / \rho}\right)}} \frac{1}{\rho}=\frac{a}{e \rho} .
\end{aligned}
$$

Hence, (24) is true.

\section{PROOF OF THEOREM 2}

Let us denote

$$
L(r)=r^{\rho^{*}(r)-\rho} .
$$

By (6) we have

$$
L(r)=\exp \left\{\vartheta_{2}(\log r)-\vartheta_{1}(\log r)\right\}
$$

We need the following amplification of (5) for a strong proximate order.

LEMMA 4.1 The following relation holds

$$
\begin{aligned}
(k r)^{\rho^{*}(k r)}-r^{\rho^{*}(r)} & =r^{\rho^{*}(r)}\left(k^{\rho}-1\right)+k^{\rho}(k-1) r^{\rho+1} L^{\prime}(r) \\
& +k^{\rho}(k-1)^{2} r^{\rho^{\rho^{*}(r)}} o(1), \quad r \rightarrow \infty,
\end{aligned}
$$

uniformly in each interval $0<a \leq k \leq b<\infty$.

Proof We have

$$
(k r)^{\rho^{*}(k r)}-r^{\rho^{*}(r)}=r^{\rho} L(r)\left(k^{\rho}-1\right)+(k r)^{\rho}[L(k r)-L(r)] .
$$

By Taylor's formula,

$$
\left|L(k r)-L(r)-(k-1) r L^{\prime}(r)\right|=\frac{1}{2}(k-1)^{2} r^{2}\left|L^{\prime \prime}(c)\right|,
$$


for some $c$ between $r$ and $k r$. Since

$$
\begin{aligned}
c^{2} L^{\prime \prime}(c)=L(c)\{ & \left\{\vartheta_{2}^{\prime}(\log c)-\vartheta_{1}^{\prime}(\log c)\right]^{2}+\left[\vartheta_{2}^{\prime \prime}(\log c)-\vartheta_{1}^{\prime \prime}(\log c)\right] \\
- & {\left.\left[\vartheta_{2}^{\prime}(\log c)-\vartheta_{1}^{\prime}(\log c)\right]\right\}, }
\end{aligned}
$$

using (7) we obtain (without loss of generality we assume $a<1<b$ )

$$
\max _{a r \leq c \leq b r} c^{2}\left|L^{\prime \prime}(c) / L(c)\right| \rightarrow 0, \quad \text { as } r \rightarrow \infty
$$

Taking into account that $L(c) / L(r) \rightarrow 1$, as $r \rightarrow \infty$ uniformly in $c$, ar $\leq c \leq b r$, we get

$$
\max _{a r \leq c \leq b r} \frac{r^{2} L^{\prime \prime}(c)}{L(r)}=\max _{a r \leq c \leq b r} \frac{r^{2}}{c^{2}} \frac{c^{2} L^{\prime \prime}(c)}{L(c)} \frac{L(c)}{L(r)} \rightarrow 0, \quad \text { as } r \rightarrow \infty,
$$

and using (27) and (29) we get (28).

We also need the following amplification of (16) for functions satisfying conditions of Theorem 2.

LeMmA 4.2 If (13) and (14) is satisfied, then

$$
K(r, f)=\sigma \rho r^{\rho^{*}(r)}+\sigma r^{\rho+1} L^{\prime}(r)+O\left(\sqrt{r^{\rho^{*}(r)} \psi(r)}\right), \quad r \rightarrow \infty .
$$

Proof Let $1 \leq k \leq 3$. Using the conditions (13) and (14), we get

$$
\begin{aligned}
& \left|[\log M(k r, f)-\log M(r, f)]-\left[\sigma(k r)^{\rho^{*}(k r)}-\sigma r^{\rho^{*}(r)}\right]\right| \\
& =|O(\psi(k r))-O(\psi(r))| \leq C \psi(r),
\end{aligned}
$$

where $\mathrm{C}$ does not depend on $k$ or $r$. Since

$$
\begin{aligned}
\log M(k r, f)-\log M(r, f) & =\int_{r}^{k r} \frac{d}{d t} \log M(t, f) d t=\int_{r}^{k r} \frac{K(t, f)}{t} d t \\
& \geq K(r, f) \int_{r}^{k r} \frac{d t}{t}=K(r, f) \log k,
\end{aligned}
$$

we obtain by (30)

$$
K(r, f) \leq \frac{1}{\log k}\left[\sigma(k r)^{\rho^{*}(k r)}-\sigma r^{\rho^{*}(r)}+C \psi(r)\right]
$$


Using Lemma 4.1, we get (uniformly in $k \in[1,3]$ )

$$
\begin{gathered}
K(r, f) \leq \frac{1}{\log k} \sigma r^{\rho^{*}(r)}\left(k^{\rho}-1\right)+\frac{1}{\log k} k^{\rho}(k-1) \sigma r^{\rho+1} L^{\prime}(r) \\
+\frac{1}{\log k} k^{\rho}(k-1)^{2} r^{\rho^{*}(r)} o(1)+\frac{1}{\log k} C \psi(r) .
\end{gathered}
$$

Using

$$
\log M(r, f)-\log M(r / k, f)=\int_{r / k}^{r} \frac{K(t, f)}{t} d t \leq K(r, f) \log k
$$

Lemma 4.1 and (30), we obtain

$$
\begin{gathered}
K(r, f) \geq \frac{1}{\log k} \sigma r^{\rho^{*}(r)}\left(1-k^{-\rho}\right)+\frac{1}{\log k} k^{-\rho}\left(1-k^{-1}\right) \sigma r^{\rho+1} L^{\prime}(r) \\
+\frac{1}{\log k} k^{-\rho}\left(1-k^{-1}\right)^{2} \sigma r^{\rho^{*}(r)} o(1)-\frac{1}{\log k} C \psi(r) .
\end{gathered}
$$

Choosing

$$
k=1+\sqrt{\psi(r) r^{-\rho^{*}(r)}},
$$

we see that the estimate in (31) will give the upper bound and the estimate in (32) will give the lower bound for $K(r, f)$.

Now we will prove Theorem 2. Let $w$ be a maximum modulus point. Following Macintyre [3] define

$$
\Psi_{w}(z):=\frac{f\left(w e^{z}\right)}{f(w)} e^{-K(|w|, f) z}
$$

Let

$$
P(h, w):=\max _{|z| \leq h}\left|\Psi_{w}(z)\right|, \quad 0<h \leq 1 .
$$

Denoting $|w|$ by $r$, we have

$$
\log \left|\Psi_{w}(z)\right| \leq \log M\left(r e^{\operatorname{Re} z}, f\right)-\log M(r, f)-K(r, f) \operatorname{Re} z .
$$

Set $\operatorname{Re} z=t$. Then

$$
\log P(h, w) \leq \max _{-h \leq t \leq h}\left(\log M\left(r e^{t}, f\right)-\log M(r, f)-K(r, f) t\right) .
$$


It is easy to see that

$$
\begin{aligned}
& \log M\left(r e^{t}, f\right)-\log M(r, f)-t K(r, f) \\
& =\int_{r}^{r e^{t}}[K(u, f)-K(r, f)] \frac{d u}{u} \\
& =\int_{r}^{r e^{t}}\left[K(u, f)-\sigma \rho u^{\rho^{*}(u)}-\sigma u^{\rho+1} L^{\prime}(u)\right]-\left[K(r, f)-\sigma \rho r^{\rho^{*}(r)}\right. \\
& \left.\quad-\sigma r^{\rho+1} L^{\prime}(r)\right] \frac{d u}{u}+\sigma \int_{r}^{r e^{t}}\left[\rho u^{\rho^{*}(u)}+u^{\rho+1} L^{\prime}(u)\right]-\left[\rho r^{\rho^{*}(r)}+r^{\rho+1} L^{\prime}(r)\right] \frac{d u}{u} .
\end{aligned}
$$

Using Lemma 4.2 and the identity

$$
r \frac{d}{d r} r^{\rho^{*}(r)}=r \frac{d}{d r} r^{\rho} L(r)=\rho r^{\rho^{*}(r)}+r^{\rho+1} L^{\prime}(r)
$$

we get

$$
\begin{aligned}
& \left|\log M\left(r e^{t}, f\right)-\log M(r, f)-t K(r, f)\right| \\
& \leq\left|\int_{r}^{r e^{t}} C_{1} \sqrt{u^{\rho^{*}(u)} \psi(u)} \frac{d u}{u}\right| \\
& \quad+\left|\int_{r}^{r e^{t}} C_{1} \sqrt{r^{\rho^{*}(r)} \psi(r)} \frac{d u}{u}\right|+\sigma\left|\left(r e^{t}\right)^{\rho^{*}\left(r e^{t}\right)}-r^{\rho^{*}(r)}-t\left[\rho r^{\rho^{*}(r)}+r^{\rho+1} L^{\prime}(r)\right]\right| \\
& =: Y_{1}+Y_{2}+\sigma Y_{3} .
\end{aligned}
$$

Let us estimate $Y_{1}, Y_{2}, Y_{3}$. Since $r^{\rho^{*}(r)} \psi(r)$ is monotonically increasing for sufficiently large $\mathrm{r}$, we have for $t<0$,

$$
Y_{1} \leq C_{1}|t| \sqrt{r^{\rho^{*}(r)} \psi(r)}
$$

Using (5) and (14), we have for $0<t \leq h<1$,

$$
Y_{1} \leq C_{1} t \sqrt{\left(r e^{t}\right)^{\rho^{*}\left(r e^{t}\right)} \psi\left(r e^{t}\right)} \leq C_{2} t \sqrt{r^{\rho^{*}(r)} \psi(r)} .
$$

Hence

$$
Y_{1} \leq C_{3}|t| \sqrt{r^{\rho^{*}(r)} \psi(r)}, \quad \text { for }|t| \leq h .
$$

Evidently,

$$
Y_{2}=C_{1}|t| \sqrt{r^{\rho^{*}(r)} \psi(r)} .
$$

Finally using Lemma 4.1, we get

$$
\begin{aligned}
Y_{3}= & \mid r^{\rho^{*}(r)}\left(e^{t \rho}-1\right)+e^{t \rho}\left(e^{t}-1\right) r^{\rho+1} L^{\prime}(r)+r^{\rho^{*}(r)} e^{t \rho}\left(e^{t}-1\right)^{2} o(1) \\
& \quad-t \rho r^{\rho^{*}(r)}-t r^{\rho+1} L^{\prime}(r) \mid \\
= & r^{\rho^{*}(r)}\left|\left(e^{t \rho}-1-\rho t\right)+\left(e^{t(1+\rho)}-e^{t \rho}-t\right) o(1)+e^{t \rho}\left(e^{t}-1\right)^{2} o(1)\right| .
\end{aligned}
$$


Since $o(1)$ 's are uniform with respect to $t,|t| \leq 1$, we obtain

$$
Y_{3} \leq C_{4} r^{r^{*}(r)} t^{2}
$$

where $C_{4}>0$ does not depend on both $r$ and $t$.

Hence, by (34) and (35)-(37) we conclude

$$
\left|\log M\left(r e^{t}, f\right)-\log M(r, f)-t K(r, f)\right| \leq D_{1}|t| \sqrt{r^{\rho^{*}(r)} \psi(r)}+D_{2} r^{\rho^{*}(r)} t^{2} .
$$

Using (33) we obtain

$$
\log P(h, w) \leq D_{1} h \sqrt{r^{\rho^{*}(r)} \psi(r)}+D_{2} r^{\rho^{*}(r)} h^{2} .
$$

Introducing

$$
\zeta_{w}(z):=\frac{P\left(\Psi_{w}(z)-1\right)}{P^{2}-\Psi_{w}(z)},
$$

we conclude similarly as we did for the function $\Phi_{w}$ in the proof of Theorem 1.(i), that

$$
\Psi_{w}(z) \neq 0 \quad \text { for }|z|<h / P \text {. }
$$

Therefore

$$
f\left(w e^{z}\right) \neq 0 \text { for }|z|<h / P<1 \text {. }
$$

This implies (remind that $r=|w|$ )

$$
R(w, f) \geq \min _{|z|=h / P}\left|w-w e^{z}\right|=r \min _{|z|=h / P}\left|1-e^{z}\right| \geq r C \frac{h}{P} .
$$

Hence, with inequality (38) we obtain

$$
R(w, f) \geq C r h \exp \left\{-\left(D_{1} h \sqrt{r^{\rho^{*}(r)} \psi(r)}+D_{2} h^{2} r^{\rho^{*}(r)}\right)\right\} .
$$

Setting $h=1 / \sqrt{r^{\rho^{*}(r)} \psi(r)}$, we get for sufficiently large values of $|w|$

$$
R(w, f) \geq C^{\prime}|w| \frac{1}{\sqrt{|w|^{\rho^{*}(|w|)} \psi(|w|)}} .
$$

This is equivalent to (15).

\section{Acknowledgement}

The authors thank the referee for suggestions that improved the presentation. 


\section{References}

[1] A.A. Goldberg, B.Ya. Levin and I.V. Ostrovskii (1996). Entire and meromorphic functions. In: R. Gamkrelidze (Ed.), Encyclopedia of Math. Sciences, Vol. 85, pp. 1-193. Springer, Berlin.

[2] W.K. Hayman (1974). The local growth of power series: a survey of the Wiman-Valiron method. Canad. Math. Bull., 17, 317-358.

[3] A.J. Macintyre (1938). Wiman's method and "flat regions" of integral functions. Quart. J. Math., 9, 81-88.

[4] B.Ya. Levin (1980). Distribution of Zeros of Entire Functions. AMS, Providence, R.I.

[5] D. Ruelle (1969). Statistical Mechanics: Rigorous Results. Addison-Wesley, New York.

[6] Sh. Strelitz (1972). Asymptotic Properties of Analytical Solutions of Differential Equations. Mintis, Vilnius (Russian). 